\title{
Implementation and analysis of polymeric microstructure replication by micro injection molding
}

\author{
Yu-Chuan $\mathrm{Su}^{1,2}$, Jatan $\mathrm{Shah}^{3}$ and Liwei $\operatorname{Lin}^{1,2}$ \\ ${ }^{1}$ Berkeley Sensor \& Actuator Center, University of California, Berkeley, CA 94720, USA \\ ${ }^{2}$ Department of Mechanical Engineering, University of California, Berkeley, CA 94720, USA \\ ${ }^{3}$ Department of Mechanical Engineering, University of Michigan, Ann Arbor, \\ MI 48105, USA \\ E-mail: yuchsu@me.berkeley.edu
}

Received 30 September 2003

Published 17 December 2003

Online at stacks.iop.org/JMM/14/415 (DOI: 10.1088/0960-1317/14/3/015)

\begin{abstract}
This paper presents the adaptation of a conventional injection molding process to the mass replication of polymeric microstructures with appropriate mold design and process control. Using wet-etched silicon wafers with microstructures on the surfaces as mold inserts, we have successfully predicted, improved and optimized the replication results. The flow behaviors of polymer melts in micro mold-cavities are characterized by both simulation and experiments. Among various process parameters, temperature is identified as the key factor that decisively determines the quality of injection-molded microstructures. Based on the collected experimental and simulation results, process optimization is performed to improve replication quality and to establish guidelines for potential applications. Because of its high speed and low cost, the adaptation of the injection molding process to microfabrication will lead to a promising technology for MEMS applications.
\end{abstract}

\section{Introduction}

Because of their unique properties, polymers have been increasingly used in a wide range of applications including both macro- and micro-devices. In order to expand the field of MEMS to polymer-based devices, it is important to introduce effective techniques for the fabrication of polymeric microstructures at a low cost and with high precision. In recent years, a number of technologies for polymeric microstructure replication have been proposed, including the LIGA process $[1,2]$ that uses either hot embossing [3] or injection molding [4] to duplicate polymeric microstructures. Using mold inserts fabricated by x-ray lithography, the LIGA process provides the possibility to manufacture microstructures with arbitrary lateral geometry and high depth for high aspect ratio devices from a variety of materials such as metals, polymers and ceramics by various molding processes. Among different molding techniques, injection molding is the most prominent one with advantages of low cost and high precision for mass production. Successful results for the replication of polymeric microstructures have been achieved by using special injection molding processes [5-12] and conventional CDinjection molding techniques [13, 14]. However, the flow behaviors of polymer melts in micro mold-cavities are not fully understood. It is believed that due to the large surface-tovolume ratio, surface effects will dominate the flow behavior at the microscale [15].

This paper aims to investigate the flow behavior of polymer melt in the micro mold-cavity and determine the necessary strategies to adapt the traditional injection molding process for the replication of polymeric microstructures. First, the direct application of the conventional injection molding process in the replication of polymeric microstructures is analyzed using a simulation software C-MOLD [16]. Different combinations of process parameters are then simulated to investigate the flow behavior of polymer melt, the relationship between process parameters and the quality of molded microstructures. Using these results, the most significant parameters can be identified and possible processing strategies can be proposed and simulated to test the feasibility. Finally, 


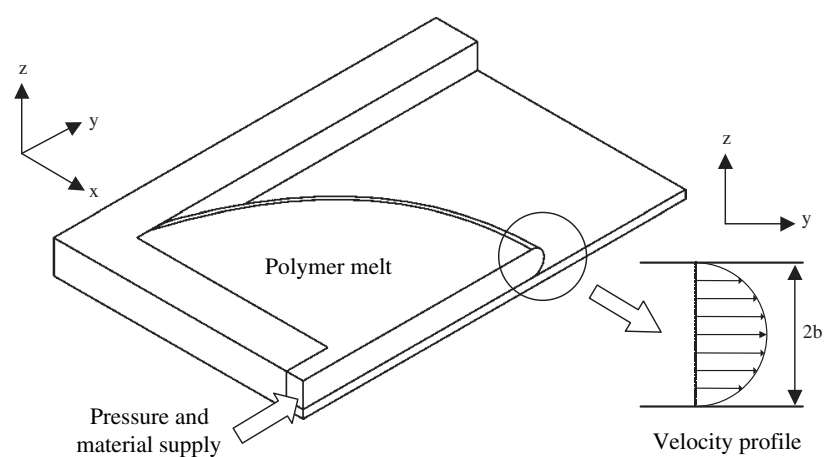

Figure 1. Schematic of polymer melt flowing in a thin cavity.

these strategies are applied in mold trials to evaluate their validity.

\section{Theoretical models}

Because most injection molded polymeric parts have complicated three-dimensional (3D) configurations and the rheological response of polymer melt is generally nonNewtonian and non-isothermal, it is extremely difficult to analyze the filling process without simplifications. The generalized Hele-Shaw (GHS) flow model introduced by Hieber and Shen [17] is the most common approximation that provides simplified governing equations for non-isothermal, non-Newtonian and inelastic flows in a thin cavity, as shown in figure 1. The assumptions of the GHS flow model are

(1) The thickness of the cavity is much smaller than the other dimensions.

(2) The velocity component in the direction of thickness is neglected, and pressure is a function of $x$ and $y$ only.

(3) The flow regions are considered to be fully developed Hele-Shaw flows in which inertia and gravitational forces are much smaller than viscous forces.

(4) The flow kinematics is shear-dominated and the shear viscosity is taken to be both temperature and shear rate dependent.

The detailed derivations have been developed by Hieber and Shen, and these assumptions apply well for the micro injection molding process. In view of these assumptions and neglecting compressibility during the filling stages, the momentum equation in the Cartesian coordinate system reduces to [17]

$$
\frac{\partial}{\partial z}\left[\eta \frac{\partial v_{x}}{\partial z}\right]=\frac{\partial P}{\partial x} \quad \frac{\partial}{\partial z}\left[\eta \frac{\partial v_{y}}{\partial z}\right]=\frac{\partial P}{\partial y}
$$

where $v_{x}$ and $v_{y}$ are velocity components in the $x$ and $y$ directions, respectively; $P(x, y)$ is the pressure, $\eta\left(\gamma^{\prime}, T\right)$ is the shear viscosity, $\gamma^{\prime}$ is the shear rate and $T$ is temperature. Under the present assumptions, $\gamma^{\prime}$ is given by

$$
\gamma^{\prime}=\left\{\left[\frac{\partial v_{x}}{\partial z}\right]^{2}+\left[\frac{\partial v_{y}}{\partial z}\right]^{2}\right\}^{1 / 2}
$$

Applying the lubrication approximation, the thicknessaveraged continuity equation results in

$$
\frac{\partial\left(b \bar{v}_{x}\right)}{\partial x}+\frac{\partial\left(b \bar{v}_{y}\right)}{\partial y}=0
$$

where $\bar{v}_{x}$ and $\bar{v}_{y}$ are averaged velocities over $z$, and $b$ is half of the thickness. After several derivative steps, the governing equation for the flow of the polymer melt can be reduced to the celebrated Reynolds equation:

$$
\frac{\partial}{\partial x}\left[S \frac{\partial P}{\partial x}\right]+\frac{\partial}{\partial y}\left[S \frac{\partial P}{\partial y}\right]=0
$$

where $S$ is the flow conductance which is defined as

$$
S=\int_{0}^{b} \frac{z^{2}}{\eta} \mathrm{d} z
$$

The velocities and shear rate can be obtained as

$$
v_{x}=\Lambda_{x} \int_{z}^{b} \frac{z_{1}}{\eta} \mathrm{d} z_{1} \quad v_{y}=\Lambda_{y} \int_{z}^{b} \frac{z_{1}}{\eta} \mathrm{d} z_{1} \quad \gamma^{\prime}=\frac{z \Lambda}{\eta}
$$

where

$$
\Lambda_{x}=-\frac{\partial P}{\partial x}, \quad \Lambda_{y}=-\frac{\partial P}{\partial y} \quad \text { and } \quad \Lambda=\left[\Lambda_{x}^{2}+\Lambda_{y}^{2}\right]^{1 / 2} .
$$

Because of the temperature difference between mold and polymer melt and the viscous heating inside the flow, the filling process should be treated as a non-isothermal case. Heat conduction in the direction of flow is neglected based on the assumption that the thickness $2 b$ is much smaller than the other two dimensions. The energy equation in the melt region becomes

$$
\rho c_{p}\left[\frac{\partial T}{\partial t}+v_{x} \frac{\partial T}{\partial x}+v_{y} \frac{\partial T}{\partial y}\right]=k \frac{\partial^{2} T}{\partial z^{2}}+\eta \gamma^{\prime 2}
$$

where the $\eta \gamma^{\prime 2}$ is the viscous heating term, and $\rho, c_{p}$ and $k$ are density, specific heat and thermal conductivity, respectively. For simplicity, it is assumed that the velocity and temperature are symmetric in the $z$ direction, the velocities of polymer melt on the mold surfaces are zero and the temperature of mold remains at $T_{w}$ during filling. The boundary conditions are given by

$$
\begin{aligned}
& v_{x}=v_{y}=0 \quad \text { at } z=b \quad \frac{\partial v_{x}}{\partial z}=\frac{\partial v_{y}}{\partial z}=0 \quad \text { at } z=0 \\
& T=T_{w} \quad \text { at } z= \pm b \quad \frac{\partial T}{\partial z}=0 \quad \text { at } z=0 .
\end{aligned}
$$

As can be seen, the equations of this model are nonlinear and coupled. It is difficult to solve these equations analytically. In this paper, simulation software C-MOLD that employs numerical solvers based on a hybrid finite element/finite difference method is used to solve the pressure, velocity and temperature fields of the GHS model. Because of these approximations, a GHS model cannot predict the exact flow field near the advancing flow front or at the edges of the mold. This might cause errors in predicting the flow behavior near microscale mold cavities.

\section{Design and fabrication of molding apparatus}

An aluminum mold is manufactured for the replication process. The schematic diagram and a photograph of the aluminum mold, which consists of cavity and core halves, are shown in figure 2. The cavity half incorporates the cavity in which a mold insert is kept. A 4-inch silicon 

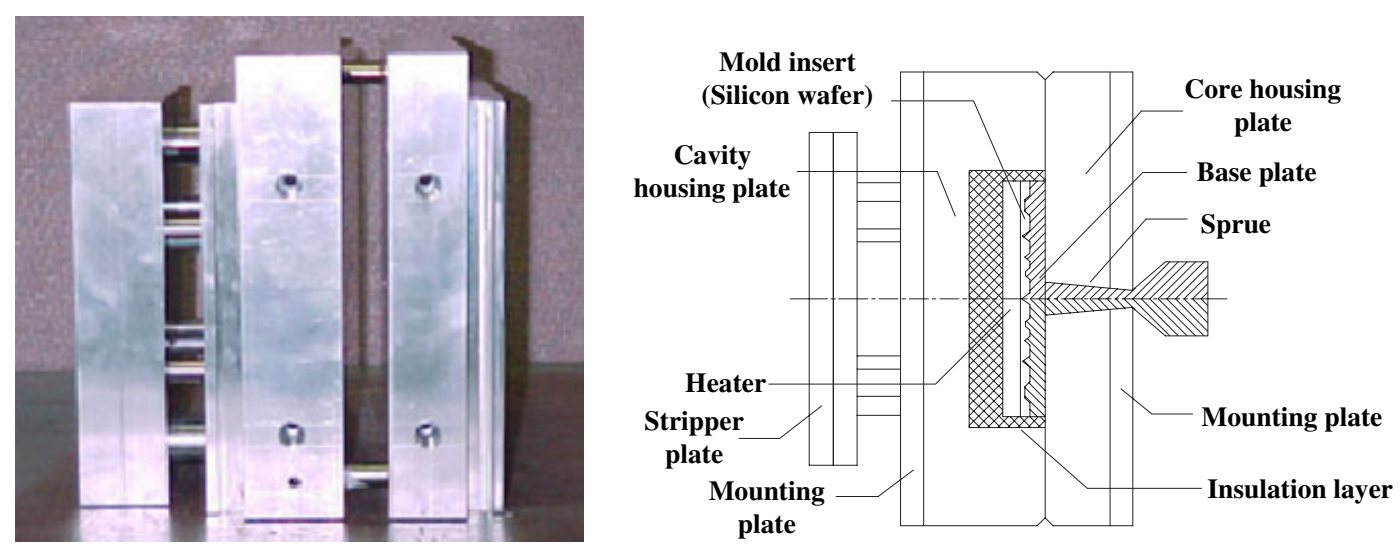

Figure 2. Injection mold set-up.

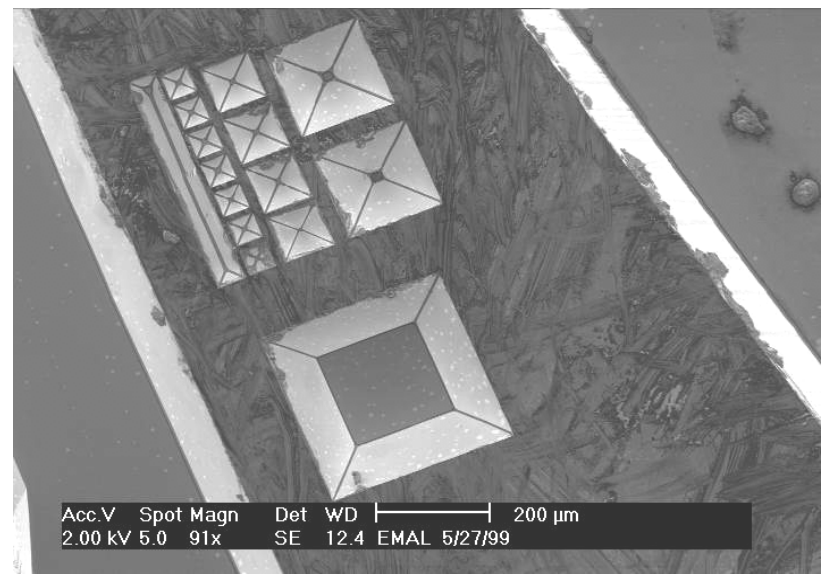

Figure 3. Microstructures on a silicon mold insert.

wafer with bulk micromachined microstructures is used as the mold insert. Figure 3 shows the silicon micro moldinsert that is etched to have a cavity depth of $110 \mu \mathrm{m}$. The square cavities have openings of $320 \mu \mathrm{m}, 160 \mu \mathrm{m}, 80 \mu \mathrm{m}$ and $40 \mu \mathrm{m}$ and are etched by means of anisotropic silicon etching in TMAH (tetramethyl-ammonium hydroxide). A heater is installed in the injection mold to control the temperature during the molding process. To have better thermal conductivity and shorter cooling time, we employed an aluminum mold that is also easier to manufacture and modify. In addition, with appropriate thermal insulation and a cooling system, the problem of dimensional variation caused by thermal expansion can be controlled and an aluminum mold can be used as a more economical tool for the replication process.

The molded component can be removed from the mold manually or by using the ejection system. Unlike the processes described in the previous literature, a silicon wafer that serves as the mold insert is placed in the mold cavity. Using silicon wafer as mold insert has the advantage of short turnaround time. In addition, the wear of a silicon mold insert is much smaller as compared to a traditional nickel tool [18]. However, a silicon mold insert is more brittle than a nickel one. To avoid the breakage of the wafer during the molding process, the edge of silicon wafer should match the cavity boundary. A gap between the mold insert and cavity can allow polymer melt to solidify within, which would eventually lift the wafer

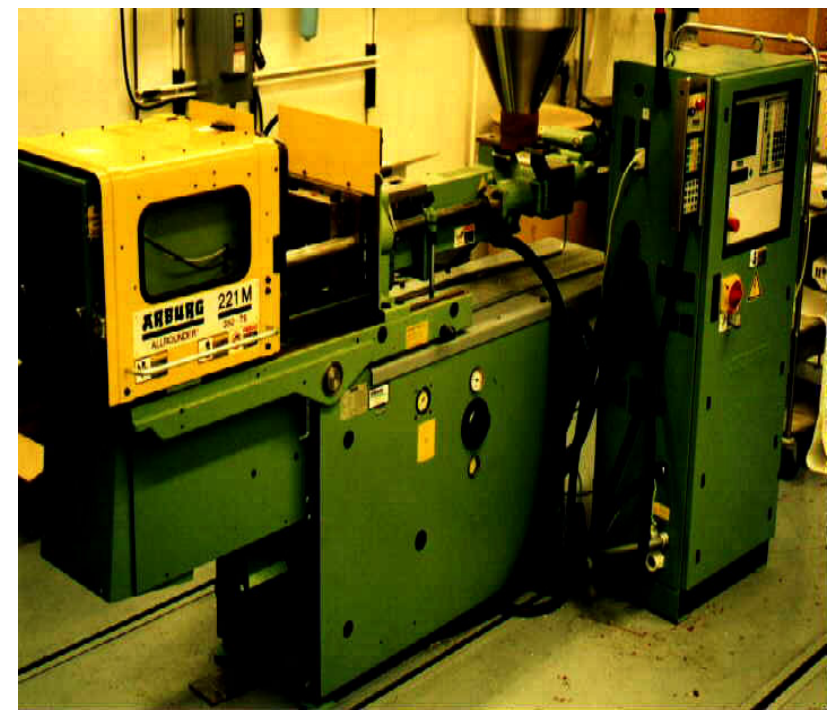

Figure 4. Arburg Allrounder 221M 350-75 injection molding machine.

from the cavity during mold opening and result in the breakage of the wafer.

\section{Experiments}

An Arburg Allrounder 221M 350-75 conventional injection molding machine, as shown in figure 4, with a single cavity, cold runner mold is employed. The material used for mold trials is Bayer Makrolon 2205 polycarbonate (PC) thermoplastic resin. Because of its excellent optical, chemical and mechanical properties, PC can be used in applications such as medical instruments, biochemical sensors and data storage systems. The polymer is injected into the mold cavity at a pressure ranging from 40 to $50 \mathrm{MPa}$. The melt temperature in the feeding zone is maintained at about $300{ }^{\circ} \mathrm{C}$. The mold temperature is controlled by a heater and maintained at a temperature lower than $200{ }^{\circ} \mathrm{C}$. The cycle time of the molding process is $65 \mathrm{~s}$, and polymer melt and mold are allowed to cool down for $30 \mathrm{~s}$ after the filling stage. Figure 5 shows the typical pressure versus time and corresponding flow rate versus time relationship of the molding process. For the micromolding process, injection pressure, mold temperature and 

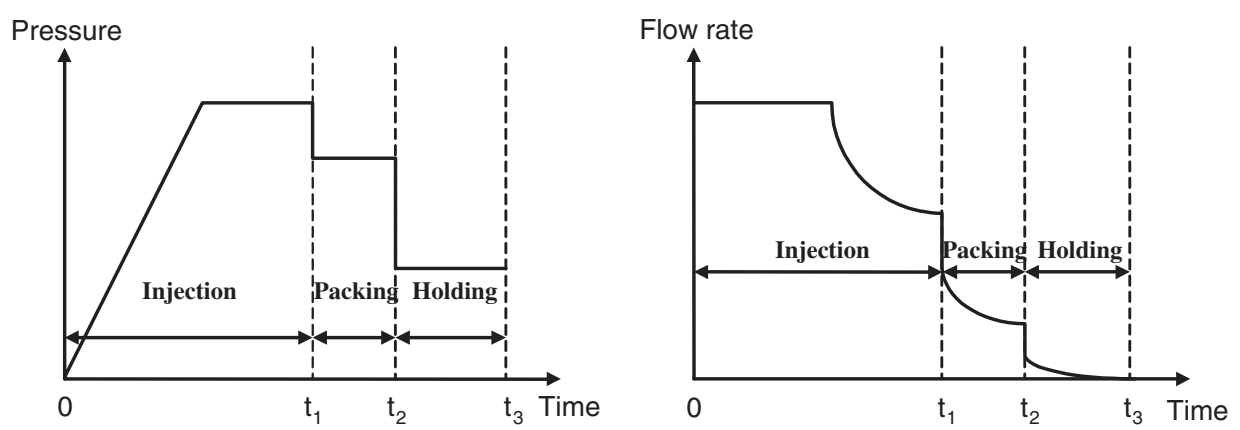

Figure 5. Typical pressure versus time and corresponding flow rate versus time relationships during the injection molding process.

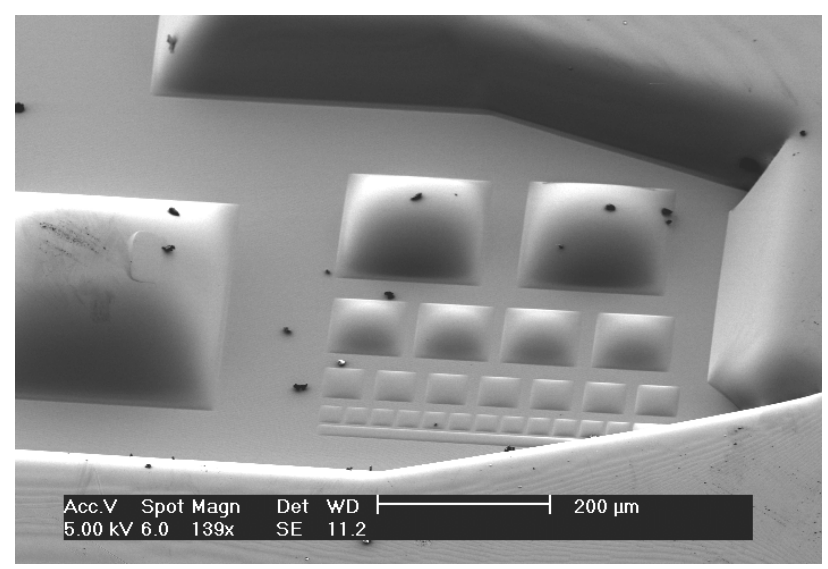

Figure 6. SEM micrograph of molding results (injection pressure $45 \mathrm{MPa}$, mold temperature $25^{\circ} \mathrm{C}$ ).

injection velocity are recognized as the driving parameters. The depth-to-opening ratios of molded microstructures are used to measure the quality of molding results. A higher depth-to-opening ratio means better filling status and molding quality.

The presence of voids plays a major role in the molding process. Preheating of the polymer prior to the molding process reduces the chances of entrapment of voids. Conventional venting methods are difficult to use for micro injection molding due to the high possibility of undesired structural changes in the molded component. Hence, an evacuated mold is recommended to obtain a good replication process.

In the first mold trial, ordinary injection molding parameters were used and no additional control unit was activated. The molding result is shown in figure 6. As can be seen, the molding results have a small depth-to-opening ratio which means polymer melt cannot fill the micro moldcavity. In this situation, polymeric microstructures cannot be successfully replicated. Before doing more mold trials to improve the molding results, a simulation tool was used to understand the flow behavior of polymer melt in the micro mold-cavity for feasible modifications to improve the molding results.

\section{Simulation}

It is well known that computer-aided engineering (CAE) can improve the trial-and-error techniques, and computer models can be relied upon to predict flow behavior and mold results. Ideally, CAE analysis provides insight that is useful in designing parts, molds and molding processes. By using CAE analysis to iterate and evaluate alternative designs and materials, engineering know-how in the form of design guidelines can be established relatively fast and costeffectively.

The CAE software C-MOLD developed by AC Technology is employed as the numerical computation tool. The mold filling process is modeled by the GHS model described in the previous section. The numerical solutions are based on a hybrid finite element/finite difference method to solve for the pressure, flow and temperature fields and a control volume method to track moving melt fronts. A finite element mesh is used to approximate the circular-shape base plate with convex microstructures on one surface, as shown in figure 7. This finite element model is composed of 6008 nodes, 2672 two-dimensional (2D) triangular elements and 4607 onedimensional (1D) part runner elements. The 2D triangular elements, which disregard the shear and cooling from side walls, are used to model the substrate plate. The 1D part runner elements, which consider the shear and cooling from all the contact surfaces, are used to model the microstructures on the surface. The following conditions are considered in this work to control and investigate the injection molding process:

- Filling time/injection pressure. In order to generate uniform molecular orientation throughout the part, it is recommended to maintain a constant velocity at the melt front. However, only advanced injection molding machines have the ability to exactly achieve this required velocity profile. In C-MOLD, either filling time or injection pressure can be used to control the process sequence.

- Mold temperature. It is believed that surface effects will dominate the flow behavior at the microscale, and melt temperatures are the key that determines the fluid property such as viscosity, specific heat and thermal conductivity. However, high temperature might cause the degradation of polycarbonate, so a pre-defined maximal allowable melt temperature is used in the simulation process [19].

- Thickness of the base plate. The base plate is employed to support microstructures and the thickness of the base plate will affect the balance of polymer melt and the quality of molded results. Because the thickness of the base plate is much larger than the individual opening of 


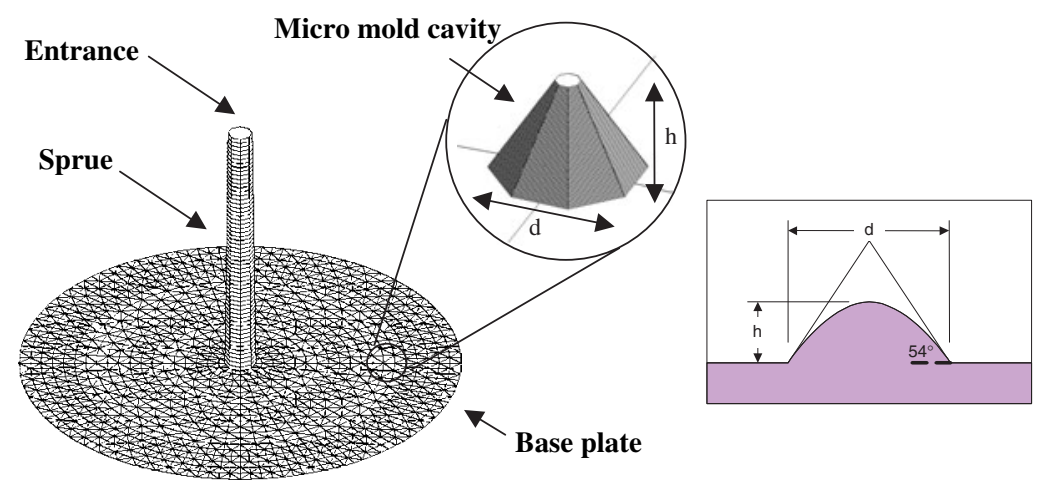

Figure 7. C-MOLD finite element model.

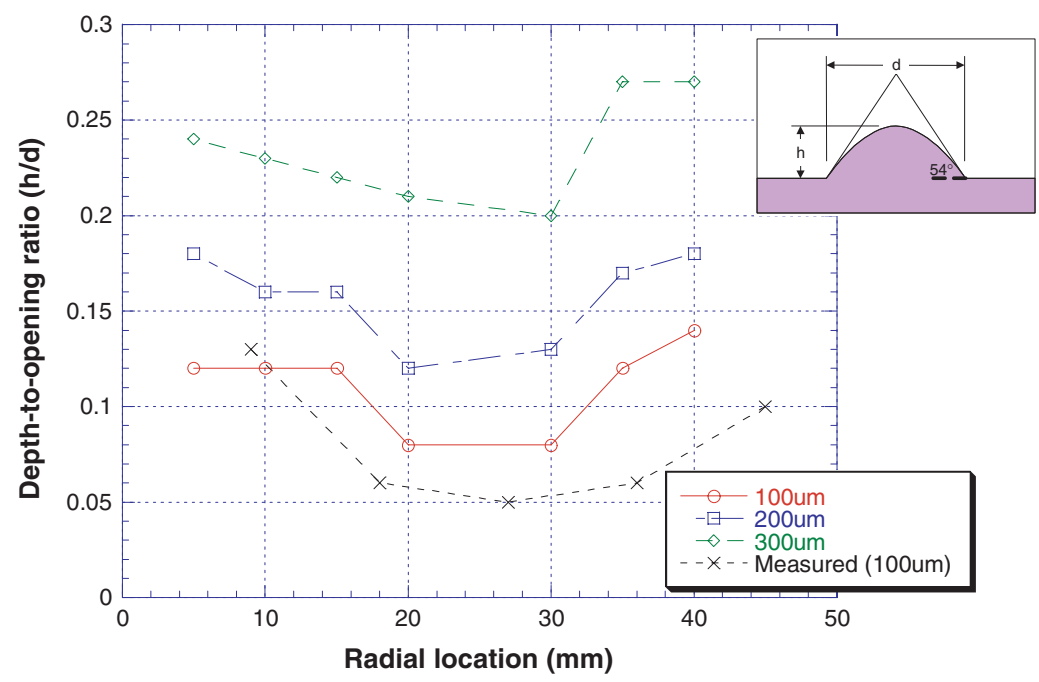

Figure 8. Simulation results of molding quality versus radial location with various cavity sizes (mold temperature $25^{\circ} \mathrm{C}$, filling time $1.5 \mathrm{~s}$ ) and measured results of $100 \mu \mathrm{m}$ cavities.

the micro mold-cavity, the problem of hesitation occurs, which requires careful consideration to control the quality of molded results.

- Depth to opening ratio. The dimensions of the micro mold-cavity determine the flow resistance and heat transfer effect. In this paper, the depth to opening ratio of the molding structures (dimensionless) is used to describe the quality of the molding process.

- Radial location. It is desirable to generate microstructures uniformly throughout the surface of the base plate. In this paper, the radial location away from the center of the base plate, which is the entering point of polymer melt, is used to investigate the quality of uniformity of the injection molding process.

Several simulation results have been obtained. Square microcavities with openings of $100 \mu \mathrm{m}, 200 \mu \mathrm{m}, 300 \mu \mathrm{m}$ and $400 \mu \mathrm{m}$ are used to investigate the injection molding process. First, a simple case is used to investigate the possibility of using traditional injection molding techniques to fabricate microstructures. In this case no cooling or heating systems are used so that the mold temperature equals ambient temperature initially. The result of this case is shown in figure 8 where a filling time of $1.5 \mathrm{~s}$ is applied. As can be seen, the depth-to-opening ratios of molded microstructures are small and not uniform. Molded microstructures in the central area have larger depth-to-opening ratios because local melt temperature and pressure close to the entrance of polymer melt are higher than other areas and facilitate better filling of the polymer melt. On the other hand, it is predicted that polymeric microstructures close to the edge also have larger depth-toopening ratios. This is believed to be the result of back pressure when the polymer melt front hits the enclosed edge of the mold cavity. If the molding process is optimized, the depthto-opening ratio should be 0.707 for cavities etched by the silicon anisotropic etching method. Generally, cavities with large openings have high penetration depth because it requires less pressure to press polymer melt into these cavities. The filling process is incomplete because insufficient material was injected into the cavity as shown. The incomplete filling may be caused by insufficient machine injection pressure (resulting from high melt resistance and a restricted flow path) or premature solidification of the polymer melt. The temperature of polymer melt in the micro mold-cavity could reduce rapidly after the polymer melt enters the cavity because of the high surface-to-volume ratio as observed in the first mold trial to 


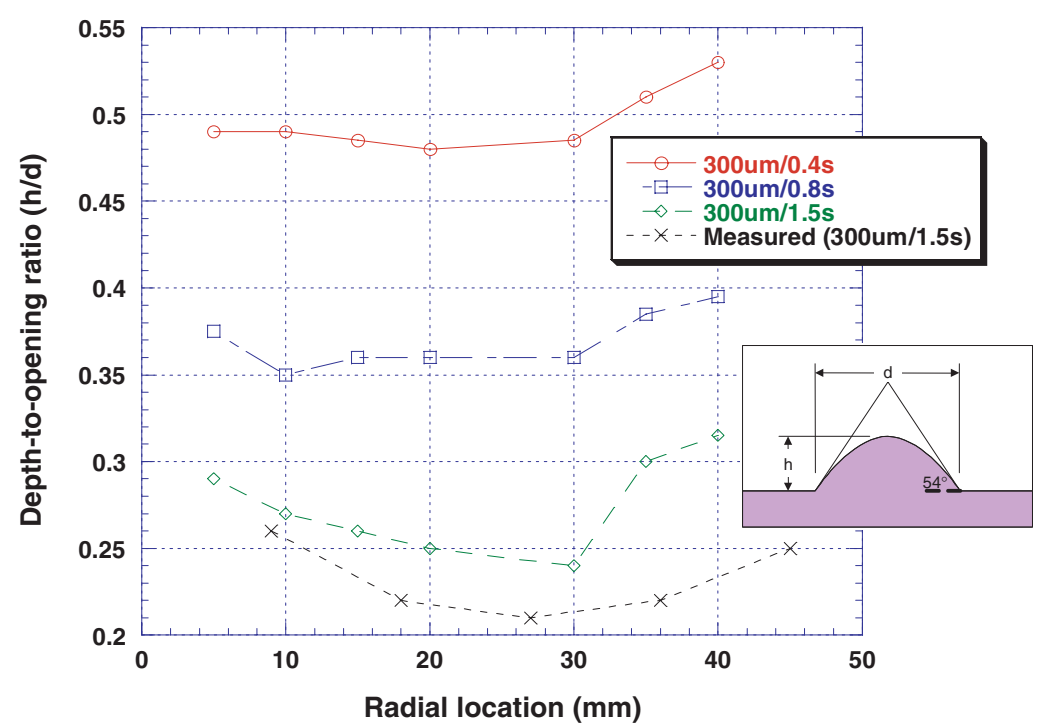

Figure 9. Simulation results of molding quality versus radial location with various filling time (mold temperature $100^{\circ} \mathrm{C}$ ) and measured results with $1.5 \mathrm{~s}$ filling time.

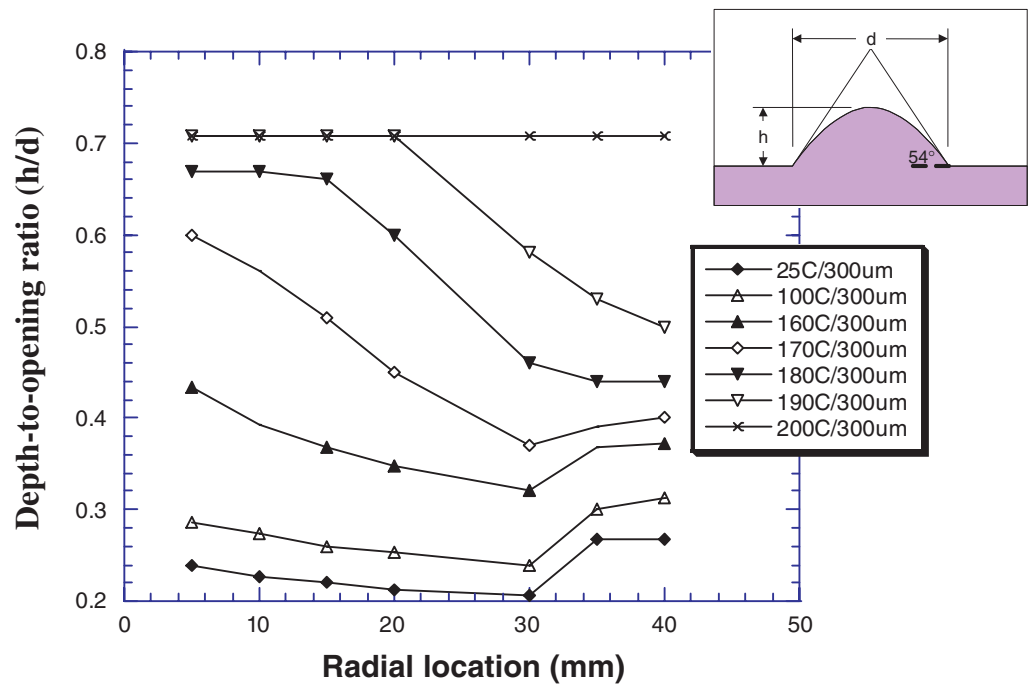

Figure 10. Simulation results of molding quality versus radial location with various mold temperature (filling time $1.5 \mathrm{~s}$ ).

cause the phenomenon of short shot. The glass transition temperature of polycarbonate is about $145^{\circ} \mathrm{C}$ and the melting temperature is $225^{\circ} \mathrm{C}$. Using a heater to keep mold cavity temperature above the glass transition temperature can help solve the solidification problems. However, it will increase the cycle time and reduce the efficiency.

Some other factors including filling time and the thickness of base plate are investigated. Figure 9 shows the simulation results between the filling time and the depth-to-opening ratios. As expected, shorter filling time, which means larger injection pressure and faster injection velocity, will generate microstructures with larger depth-to-opening ratios. The other possible modification is to change the thickness of the base plate. Based on simulation results, high injection pressure is required to push polymer melt if the thickness of the base plate is small. This modification still fails to make perfect replication. The next step to improve the molding process is to increase the molding temperature. Figure 10 shows the simulation results between mold temperature and depth-to-opening ratios when filling time is kept constant at $1.5 \mathrm{~s}$. As can been seen, the increase of mold temperature does not increase the depth-to-opening ratios dramatically if the mold temperature is low or just a little higher than the glass transition temperature. When mold temperature is at $200{ }^{\circ} \mathrm{C}$, polymer melt can fill the micromold cavity completely in $1.5 \mathrm{~s}$ as shown in the simulation results. Ideally, if mold temperature exceeds the glass transition temperature of the polymer, no solidified layer will be generated and polymer melt can fill the micromold cavity completely if suitable injection pressure and filling time are applied. However, because the viscosity of polymer melt is relatively large when its temperature is just above the glass transition temperature, the required injection pressure is extremely large or the filling time is extremely long. According to the simulation results, a mold temperature $30{ }^{\circ} \mathrm{C}$ to $40{ }^{\circ} \mathrm{C}$ higher than the glass transition temperature is recommended for the replication process. Also shown 


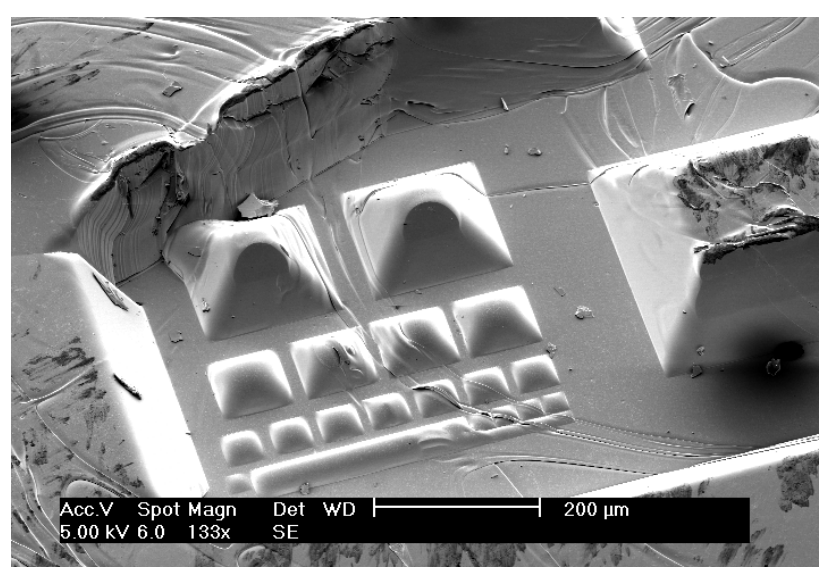

Figure 11. SEM micrograph of molding results (injection pressure $45 \mathrm{MPa}$, mold temperature $100{ }^{\circ} \mathrm{C}$ ).

in the simulation results, the improvement on the molding results in the area close to the edge is less than those in other areas. Because of the significant decrease of viscosity at higher mold temperature, the required pressure for driving polymer melt into the micro-cavity is significantly reduced. Once the mold temperature exceeds a certain limit, a polymer melt will be able to fill all micro mold-cavities on the surface. As the temperature becomes the dominant factor, the issue of back pressure becomes less important. It is believed that the temperature far away from the center of the base plate will be lower compared to the central region. As a result, the depth-toopening ratio becomes smaller as indicated in the simulation results.

When mold temperature is higher than the glass transition temperature of the polymer, the analysis is simplified by using an isothermal model which assumes that the polymer melt temperature is fixed at the mold temperature during the filling stage. This isothermal assumption is verified by a non-isothermal model, and the simulation results show that the difference between these two models is less than $10 \%$ in the worse case in predicting the depth-to-opening ratio. Consequently, the energy equation can be omitted and the flow behavior can be obtained by solving two nonlinear and coupled equations instead of three.

\section{Experiment results and discussions}

As discussed previously, a temperature control unit was found to be most effective in achieving good molding results. Therefore, a heater capable of maintaining mold temperature was mounted on the backside of the mold insert to control the mold temperature. Figure 11 shows the results of mold trials after the heater is set at $100{ }^{\circ} \mathrm{C}$. As can be seen, large microstructures (with openings larger than $100 \mu \mathrm{m}$ ) have flat instead of curved surfaces which means that the polymer melt can flow deeper into the micro mold-cavity and replicate the pyramidal shape of the cavity. On the other hand, small microstructures (with openings smaller than $100 \mu \mathrm{m}$ ) have better (but still curved) replication results as compared to the previous results. The depth-to-opening ratios

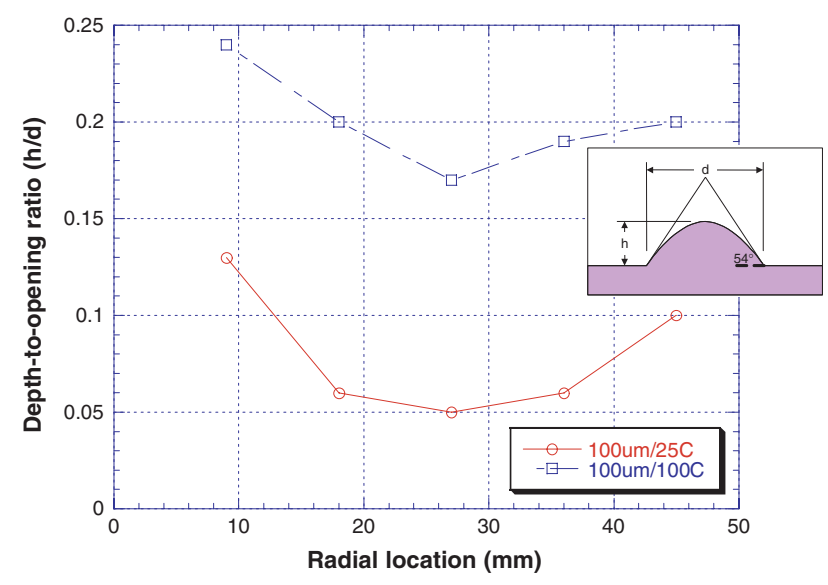

Figure 12. Measurement results of molding quality versus radial location with various mold temperature (injection presssure $45 \mathrm{MPa})$.

of replicated microstructures are measured using a whitelight interferometer (Wyko NT 3300 profiling system) and the results are shown in figures 8,9 and 12. The comparison of figures 8 and 9 indicates that the general trend, having higher depth-to-opening ratio around the center and edge, is predicted by the simulation results with $20 \%$ to $40 \%$ overestimation probably due to temperature variation across the mold. Figure 12 compares the measured depth-to-opening ratio against radial location at $25^{\circ} \mathrm{C}$ and $100{ }^{\circ} \mathrm{C}$ and verifies the effects caused by raising mold temperature as simulation results suggest.

As predicted by simulation results, polymer melt cannot fill the micro mold-cavity completely. Based on figure 10, the mold temperature should be higher than $200{ }^{\circ} \mathrm{C}$ for complete duplication. However, in this prototype demonstration, the heating strip used is unable to maintain such a high temperature. Therefore, one of the future works is to have a more sophisticated heating device and mold design to achieve a higher mold temperature for optimized molding results. On the other hand, the high mold temperature will also increase the required cooling period. A cooling system can bring down the overall temperature effectively and reduce the cooling time. Another problem is air bubbles trapped in molded polymer structures. In conventional molding processes, air vent slots facilitate the escape of the air in the mold during mold filling. However, these slots are close to the size of the microstructures so they will be filled by the adapted micro-molding process and fail to vent the trapped air. Therefore, it is often suggested that an evacuation system should be constructed for the micro injection molding process.

\section{Conclusions}

A conventional injection molding technique for the replication of polymeric microstructures is investigated. According to the simulation and experimental results, a conventional injection molding process with a specific process control strategy can be applied in the fabrication of microstructures. Because of the large surface-to-volume ratio of the micromold cavity, the temperature of the polymer melt reduces rapidly right after it 
reaches the entrance of the cavity. If the mold temperature is lower than the transition temperature of polymer, boundary solidification will prevent the melt from flowing into the cavity. This phenomenon contributes to the low depth-to-opening ratio in the preliminary molding results. Two modifications have been identified to improve the process. First, the mold temperature must remain much higher than the transition temperature of polymer to reduce the viscosity during the filling stage. In this situation, no solidified layer will be generated and longer filling time and lower injection pressure can be employed to reduce residual stress. Secondly, a vacuum is preferred in the micro mold-cavity to avoid possible air traps. In addition, parameters such as injection velocity, injection pressure, holding pressure, cooling time and melt temperature must also be controlled appropriately, based on the mold designs, to achieve optimal performance.

\section{Acknowledgments}

This work is supported in part by a NSF award (DMI-9800434) and a DARPA/MTO/MEMS grant. The author would like to thank Mr Kent Pruss for assisting the manufacturing of aluminum mold and Mr Gerald Polashak for operating the injection molding machine.

\section{References}

[1] Menz W, Bacher W, Harmening M and Michel A 1991 The LIGA technique-a novel concept for microstructures and the combination with Si-technologies by injection molding Proc. IEEE Micro Electro Mechanical Systems Workshop pp 69-73

[2] Bacher W, Menz W and Mohr J 1995 The LIGA technique and its potential for microsystems - a survey IEEE Trans. Ind. Electron. 42 431-41

[3] Both A, Bacher W, Heckele M, Muller K D, Ruprecht R and Strohmann M 1995 Molding process with high alignment precision for the LIGA-technology Proc. IEEE Micro Electro Mechanical Systems Conference pp 186-90

[4] Ruprecht R, Hanemann T, Piotter V and Hausselt J 1995 Injection molding of LIGA and LIGA-similar microstructures using filled and unfilled thermo-plastics Proc. SPIE 2639 146-57
[5] Weber L, Ehrfeld W, Freimuth H, Lacher M, Lehr H and Pech B 1996 Micromolding - a powerful tool for the large scale production of precise microstructures Proc. SPIE 2879 156-67

[6] Rogalla A and Michaeli W 1997 Analysis of injection molded microstructures Proc. SPE 55th Annual Technical Conference pp 365-8

[7] Piotter V, Hanemann T, Ruprecht R, Thies A and Hausselt J 1997 New developments of process technologies for microfabrication Proc. SPIE 3223 91-9

[8] Despa M S, Kelly K W and Collier J R 1999 Injection molding of polymeric LIGA HARMs Microsyst. Technol. 6 60-6

[9] Piotter V, Bauer W, Benzler T and Emde A 2001 Injection molding of components for microsystems Microsyst. Technol. 7 99-102

[10] Piotter V, Mueller K, Plewa K, Ruprecht R and Hausselt J 2002 Performance and simulation of thermoplastic microinjection molding Microsyst. Technol. 8 pp 387-90

[11] Madou M J, Lee L J, Koelling K W, Daunert S, Lai S, Koh C G, Juang Y-J, Yu L and Lu Y 2001 Design and fabrication of polymer microfluidic platforms for biomedical applications Proc. SPE 59th Annual Technical Conference pp 2534-38

[12] Yu L, Koh C G, Lee L J, Koelling K W and Madou M J 2002 Experimental investigation and numerical simulation of injection molding with micro-features Polym. Eng. Sci. 42 $871-88$

[13] Larsson O, Ohman O, Billman A, Lundbladh L, Lindell C and Palmskog G 1997 Silicon based replication technology of 3D-microstructures by conventional CD-injection molding techniques Proc. Int. Conf. on Solid-State Sensors and Actuators pp 1415-8

[14] Ohman O 2000 Polymer replication of microfluidic CD devices Proc. 2nd Annual Int. Conf. on Manufacturing \& Commercialization Issues for Micro \& Nano Medical Devices

[15] Ho C-M and Tai Y-C 1998 Micro-electro-mechanical-systems (MEMS) and fluid flows Annu. Rev. Fluid Mech. 30 579-612

[16] AC Technology 1998 C-MOLD version 4.0

[17] Hieber C A and Shen S F 1982 A finite-element/ finite-different simulation of the injection-molding filling process J. Non-Newtonian Fluid Mech. 7 1-32

[18] Becker H and Heim U 1999 Silicon as tool material for polymer hot embossing Proc. IEEE Micro Electro Mechanical Systems Conference pp 228-31

[19] Bottenbruch L 1996 Engineering Thermoplastics: Polycarbonates, Polyacetals, and Polyesters (Cincinnatti: Hanser Gardner) 